\title{
Résumé des recommandations mises à jour du Comité consultatif national de l'immunisation sur les vaccins contre le virus du papillome humain (VPH) : Intervalles minimaux entre les doses du vaccin nonavalent contre le VPH dans le calendrier d'immunisation contre le VPH
}

\author{
Tunis $\mathrm{MC}^{1}$ et Deeks $\mathrm{SL}^{2,3}$ au nom du Comité consultatif national de l'immunisation (CCNI)*
}

\section{Résumé}

Contexte : Les infections par le virus du papillome humain (VPH) sont les infections transmissibles sexuellement les plus répandues et, en l'absence de vaccination, on estime que $75 \%$ des Canadiens et Canadiennes sexuellement actifs auront une infection au VPH au cours de leur vie. Les vaccins quadrivalent (VPH4) et bivalent (VPH2) sont homologués au Canada depuis 2007 et 2010, respectivement. Le Comité consultatif national de l'immunisation (CCNI) du Canada recommandait auparavant l'immunisation par le vaccin VPH4 chez les hommes et les femmes selon un calendrier à trois doses (à 0,2 et 6 mois) ou à deux doses (à 0 et 6 mois), ou par le vaccin VPH2 chez les femmes selon un calendrier à trois doses (à 0,1 et 6 mois) ou à deux doses

(à 0 et 6 mois), en fonction de l'âge et de l'état de santé du sujet vacciné. En février 2015, un vaccin nonavalent (Gardasil@9, Merck Canada Inc.) a été homologué au Canada pour la prévention des cancers et des verrues anogénitales associés aux types $6,11,16,18,31,33,45,52$ et 58 du VPH chez les filles et les femmes âgées de 9 à 45 ans et chez les garçons et les hommes âgés de 9 à 26 ans.

Objectifs : Résumer les données probantes sur le nouveau vaccin VPH9 et formuler des recommandations pour son utilisation au Canada, examiner les données épidémiologiques sur la contribution relative aux résultats de la maladie des cinq génotypes supplémentaires contenus dans le vaccin VPH9, et clarifier les intervalles minimaux acceptables entre les doses de vaccin dans un calendrier d'immunisation à deux doses ou à trois doses.

Méthodologie : Le groupe de travail sur le VPH du CCNI a effectué des examens documentaires au sujet des intervalles minimaux entre les doses des vaccins contre le VPH ainsi que sur le vaccin VPH9. Les fabricants des vaccins ont fourni des données supplémentaires aux fins d'examen. Toutes les données probantes ont été examinées et évaluées et un ensemble de données représentatives pour chaque essai a été reporté dans des tableaux de données. Une synthèse des connaissances a été réalisée, et le $\mathrm{CCNI}$ a approuvé des recommandations précises fondées sur des données probantes, clarifiant le raisonnement et les considérations pertinentes.

Résultats: Au moment de l'examen, il n'existait qu'une seule étude revue par les pairs publiée sur le vaccin VPH9 pouvant être incluse dans l'examen, mais le fabricant du vaccin à évaluer a communiqué des renseignements d'autres études non publiées sous forme de présentations, d'affiches et de résumés.

D'après les données probantes disponibles à ce jour, le vaccin VPH9 est recommandé selon un calendrier à trois doses chez les filles et les femmes âgées de 9 à 26 ans, chez les femmes âgées de plus de 26 ans qui n'ont pas été vaccinées auparavant ou qui n'ont pas terminé la série de vaccination, et chez les garçons et les hommes âgés de 9 à 26 ans. Pour le moment, les données probantes sont insuffisantes pour recommander un calendrier d'immunisation à deux doses par le vaccin VPH9, mais un essai clinique visant à évaluer d'autres calendriers

\section{Affiliations}

${ }^{1}$ Centre de l'immunisation et des maladies respiratoires infectieuses, Agence de la santé publique du Canada, Ottawa (Ontario)

${ }^{2}$ Président du Groupe de travail du CCNI sur le VPH, Toronto (Ontario)

${ }^{3}$ Immunisation et maladies évitables par la vaccination, Santé publique Ontario, Toronto (Ontario)

*Correspondance : naci-ccni@ phac-aspc.gc.ca 
d'administration du vaccin VPH9 est actuellement en cours. On n'a pas évalué l'efficacité du vaccin VPH9 en quant à la prévention des infections et des maladies liées aux types 31, 33, 45, 52 et 58 du VPH chez les personnes ayant déjà reçu le vaccin VPH4. Au Canada, I'immunisation contre les types 16 et 18 du VPH par le vaccin VPH2, VPH4 ou VPH9 peut prévenir environ $70 \%$ des cancers anogénitaux et $60 \%$ des lésions précancéreuses du col de l'utérus à risque élevé. L'immunisation par un vaccin VPH4 ou VPH9 peut prévenir environ $90 \%$ des verrues anogénitales (types 6 et 11 du VPH). L'immunisation par le vaccin VPH9 peut prévenir jusqu'à $14 \%$ de plus des cancers anogénitaux et jusqu'à $30 \%$ de plus des lésions précancéreuses du col de l'utérus à risque élevé causés par les cinq types de VPH supplémentaires (31, 33, 45, 52 et 58) contre lesquels le vaccin fournit une protection. Le fardeau de la maladie associé aux cinq génotypes supplémentaires contenus dans le vaccin VPH9 n'est pas partagé équitablement entre les sexes, l'avantage supplémentaire étant principalement observé chez les femmes.

En ce qui concerne le calendrier d'immunisation contre le VPH, peu de données probantes appuient des intervalles minimaux souples ou raccourcis pour les vaccins contre le VPH, comparativement aux nombreuses données probantes appuyant les calendriers recommandés, ainsi qu'aux données probantes à l'appui des retards dans la réception des doses de rappel. Les hypothèses en matière d'immunogénicité et d'efficacité des « intervalles souples » raccourcis entre les doses reposent largement sur les données non publiées au dossier du fabricant et ses recommandations approuvées par Santé Canada comprises dans les monographies de produit. Les recommandations et les catégories de données probantes du CCNI fondées sur ces résultats sont présentées cidessous.

Conclusions: Outre les souches 6, 11, 16 et 18 du VPH qui peuvent être couvertes par les autres vaccins contre le VPH, le vaccin VPH9 est censé procurer une protection supplémentaire par la prévention des infections et des maladies associées aux types 31, 33, 45, 52 et 58 du VPH. La protection contre ces souches supplémentaires peut prévenir jusqu'à $14 \%$ de plus des cancers anogénitaux et jusqu'à $30 \%$ de plus des lésions précancéreuses du col de l'utérus à risque élevé au Canada. Des efforts devraient être faits pour administrer les vaccins contre le VPH aux intervalles recommandés. Si un calendrier raccourci est nécessaire, les intervalles minimaux entre les doses du vaccin contre le VPH doivent être respectés, notamment un intervalle minimal de 24 semaines entre la première et la dernière dose dans un calendrier d'immunisation à deux doses ou à trois doses. Veuillez noter que le CCNI examine actuellement les données probantes disponibles pour un calendrier d'immunisation contre le vaccin VPH à 2-doses.

Citation proposée : Tunis MC, Deeks SL au nom du Comité consultatif national de l'immunisation. Résumé des recommandations mises à jour du Comité consultatif national de l'immunisation sur les vaccins contre le virus du papillome humain (VPH) : Intervalles minimaux entre les doses du vaccin nonavalent contre le virus du papillome humain dans le calendrier d'immunisation contre le VPH. Relevé des maladies transmissibles au Canada 2016;42:166-9. https://doi.org/10.14745/ccdr.v42i07a03f

\section{Introduction}

L'examen documentaire sur les vaccins contre le virus du papillome humain (VPH) et sur le vaccin nonavalent contre le VPH ainsi que les recommandations actuelles du Comité consultatif national de l'immunisation (CCNI) sur le vaccin contre le VPH sont publiés dans la déclaration complète du CCNI (1) et dans le chapitre du Guide canadien d'immunisation consacré au VPH (2).

\section{Recommandation no 1}

Le CCNI conclut que I'un ou l'autre des vaccins contre le VPH actuellement autorisés au Canada peut être utilisé selon le calendrier d'immunisation contre le VPH recommandé Recommandation du CCNI de catégorie A ou B d'après les données probantes (tableau 1).

L'immunisation contre le VPH peut être complétée avec le vaccin VPH2, VPH4 ou VPH9 chez les femmes et avec les vaccins VPH4 ou VPH9 chez les hommes, selon les calendriers d'immunisation résumés dans le tableau 1 ci-dessous. Dans la mesure du possible, le même vaccin doit être utilisé pour compléter la série de vaccins. Si l'administration de la série vaccinale avec le même vaccin n'est pas possible, les vaccins VPH2, VPH4 ou VPH9 peuvent être utilisés pour compléter la série chez les femmes, et les vaccins VPH4 ou VPH9 peuvent être utilisés pour compléter la série chez les hommes. Le vaccin VPH9 administré à des personnes immunocompétentes âgées de 9 à 26 ans devrait offrir une protection d'efficacité semblable contre les génotypes contenus dans le vaccin VPH4. Les vaccins VPH2, VPH4 et VPH9 offrent tous une protection contre les types 16 et 18 du VPH, qui sont responsables d'environ $70 \%$ des cancers anogénitaux.

En outre, le vaccin VPH9 offre une protection contre les cinq types supplémentaires du VPH non contenus dans le vaccin VPH4 (types 31, 33, 45, 52 et 58 du VPH).

Le vaccin VPH9 offre une protection contre 5 autres génotypes du VPH responsables d'environ $14 \%$ des cancers anogénitaux. Les vaccins VPH4 et VPH9 offrent également une protection contre les génotypes 6 et 11 du VPH, qui causent plus de 
Tableau 1 : Calendrier recommandé d'immunisation par les vaccins contre le virus du papillome humain

\begin{tabular}{|c|c|c|}
\hline $\begin{array}{l}\text { Groupes } \\
\text { recommandés }\end{array}$ & $\begin{array}{l}\text { Calendrier } \\
\text { d'immunisation } \\
\text { recommandé }\end{array}$ & $\begin{array}{l}\text { Vaccin(s) et } \\
\text { catégorie } \\
\text { de données } \\
\text { probantes du } \\
\text { CCNI }\end{array}$ \\
\hline \multirow{2}{*}{$\begin{array}{l}\text { Filles en santé } \\
\text { âgées de } 9 \text { à } 14 \text { ans } \\
\text { (immunocompétentes } \\
\text { et non infectées par } \\
\text { le VIH) (et femmes en } \\
\text { santé âgées de } 15 \text { ans } \\
\text { et plus chez qui la } \\
\text { première dose a été } \\
\text { administrée entre } 9 \\
\text { et } 14 \text { ans) }\end{array}$} & $\begin{array}{l}\text { Calendrier à deux } \\
\text { ou trois doses }\end{array}$ & $\begin{array}{l}\text { VPH2 ou VPH4 } \\
\text { (Catégorie A) }\end{array}$ \\
\hline & $\begin{array}{l}\text { Calendrier à } \\
\text { trois doses }\end{array}$ & $\begin{array}{l}\text { VPH9 } \\
\text { (Catégorie B) }\end{array}$ \\
\hline $\begin{array}{l}\text { Femmes en santé } \\
\text { âgées de } 15 \text { ans et plus } \\
\text { (immunocompétentes } \\
\text { et non infectées par } \\
\text { le VIH) }\end{array}$ & $\begin{array}{l}\text { Calendrier à } \\
\text { trois doses }\end{array}$ & $\begin{array}{l}\text { VPH2 ou VPH4 } \\
\text { (catégorie A) ou } \\
\text { VPH9 (catégorie B) }\end{array}$ \\
\hline \multirow{2}{*}{$\begin{array}{l}\text { Garçons en santé } \\
\text { âgés de } 9 \text { à } 14 \text { ans } \\
\text { (immunocompétents et } \\
\text { non infectés par le VIH) } \\
\text { (et hommes en santé } \\
\text { âgés de } 15 \text { ans et plus } \\
\text { chez qui la première } \\
\text { dose a été administrée } \\
\text { entre } 9 \text { et } 14 \text { ans) }\end{array}$} & $\begin{array}{l}\text { Calendrier à deux } \\
\text { ou trois doses }\end{array}$ & VPH4 (catégorie B) \\
\hline & $\begin{array}{l}\text { Calendrier à } \\
\text { trois doses }\end{array}$ & VPH9 (catégorie B) \\
\hline $\begin{array}{l}\text { Hommes en santé } \\
\text { âgés de } 15 \text { ans et plus } \\
\text { (immunocompétents et } \\
\text { non infectés par le VIH) }\end{array}$ & $\begin{array}{l}\text { Calendrier à } \\
\text { trois doses }\end{array}$ & $\begin{array}{l}\text { VPH4 ou VPH9 } \\
\text { (catégorie B) }\end{array}$ \\
\hline $\begin{array}{l}\text { Personnes } \\
\text { immunodéprimées } \\
\text { et personnes } \\
\text { immunocompétentes } \\
\text { infectées par le VIH }\end{array}$ & $\begin{array}{l}\text { Calendrier à } \\
\text { trois doses }\end{array}$ & $\begin{array}{l}\text { VPH2, VPH4 ou VPH9 } \\
\text { chez les femmes; } \\
\text { VPH4 ou VPH9 chez } \\
\text { les hommes } \\
\text { Catégorie I }\end{array}$ \\
\hline
\end{tabular}

Abréviations : VPH, virus du papillome humain; $\mathrm{VIH}$, virus de l'immunodéficience humaine

$90 \%$ des verrues anogénitales. À l'échelle de la population, si toutes les personnes pour lesquelles le vaccin est recommandé le reçoivent et si l'efficacité du vaccin à long terme est de $100 \%$, l'immunisation par le vaccin VPH9 au Canada peut potentiellement prévenir chaque année jusqu'à 320 cas supplémentaires de cancers anogénitaux ( 300 chez les femmes et 20 chez les hommes). Les événements indésirables suivant l'immunisation avec des vaccins contre le VPH comprennent principalement la douleur, l'enflure et l'érythème légers à modérés liés au site d'injection. Ces événements indésirables locaux sont plus fréquents chez les personnes ayant reçu le vaccin VPH9 comparativement à celles ayant reçu le vaccin VPH4.

Le CCNI réévaluera la classification de cette recommandation à mesure que de nouvelles données probantes font surface.

\section{Recommandation no 2}

Le CCNI conclut que les données probantes sont actuellement insuffisantes pour recommander un calendrier d'immunisation à deux doses avec le vaccin VPH9 - Recommandation du CCNI de catégorie I d'après les données probantes.

Un essai clinique de phase III visant à étudier l'innocuité et l'immunogénicité d'un calendrier d'immunisation à deux doses avec le vaccin VPH9 est en cours. L'objectif de l'étude d'une durée de 37 mois est d'établir si les calendriers expérimentaux à deux doses à 0 et 6 mois et à 0 et 12 mois chez les filles et les garçons âgés de 9 à 14 ans sont sécuritaires et immunogènes, avec une réponse anticorps non inférieure à celle observée chez les filles et les femmes âgées de 9 à 26 ans qui ont reçu le vaccin selon le calendrier d'immunisation normalisé à trois doses.

Le $\mathrm{CCNI}$ examinera et réévaluera cette recommandation à mesure que de nouvelles données probantes font surface.

\section{Recommandation no 3}

Le CCNI conclut que les données probantes sont actuellement insuffisantes pour recommander, à l'échelle de la population, une nouvelle immunisation avec le vaccin VPH9 des personnes qui ont terminé une série vaccinale avec un autre vaccin contre le VPH - Recommandation du CCNI de catégorie I d'après les données probantes.

Les données non publiées semblent indiquer qu'une nouvelle immunisation par le vaccin VPH9 après la fin d'une série avec le vaccin VPH4 produit une plus faible immunogénicité pour les cinq autres génotypes du VPH (portée clinique inconnue) ainsi qu'une fréquence plus élevée des événements indésirables locaux au site d'injection; l'efficacité n'a pas été évaluée.

Bien qu'elle ne soit pas recommandée à l'échelle de la population, les personnes ayant reçu le vaccin VPH4 qui souhaitent tirer profit de la protection supplémentaire offerte par le vaccin VPH9 peuvent être vaccinées avec le vaccin VPH9. Les données probantes sont actuellement insuffisantes pour déterminer si l'administration de moins de trois doses de vaccin VPH9 protège contre les cinq autres types de VPH chez les sujets ayant reçu le vaccin VPH4.

Le $\mathrm{CCNI}$ examinera et réévaluera cette recommandation à mesure que de nouvelles données probantes font surface.

\section{Recommandation no 4}

Le CCNI conclut qu'il existe des données probantes solides à l'appui d'un intervalle minimal de 24 semaines (six mois) entre la première et la dernière dose dans un calendrier d'immunisation contre le VPH à deux ou trois doses Recommandation du CCNI de catégorie $A$ d'après les données probantes.

Le CCNI recommande que soient respectés, dans la mesure du possible, les intervalles recommandés entre les doses du vaccin $\mathrm{VPH} 2(0,1$ et 6 mois dans un calendrier à trois doses ou 0 et 6 mois dans un calendrier à deux doses), du vaccin VPH4 $(0,2$ et 6 mois dans un calendrier à trois doses ou 0 et 6 ou 12 mois dans un calendrier à deux doses) et du vaccin VPH9 (0, 2 et 6 mois). Lorsqu'un calendrier abrégé est inévitable, dans un calendrier à trois doses, l'intervalle minimal 
entre la première et la deuxième dose du vaccin contre le VPH est de 4 semaines (1 mois), l'intervalle minimal entre la deuxième et la troisième dose du vaccin contre le VPH est de 12 semaines ( 3 mois), et l'intervalle minimal entre la première et la troisième dose est de 24 semaines ( 6 mois), tels qu'ils sont résumés dans le tableau 1 . L'intervalle minimal entre la première et la deuxième dose d'un calendrier à deux doses avec le vaccin VPH2 ou VPH4 est de 24 semaines (6 mois).

\section{Conflit d'intérêts}

Aucun.

\section{Financement}

Les travaux du CCNI sont appuyés par l'Agence de la santé publique du Canada.

\section{Références}

1. Comité consultatif national de l'immunisation. Recommandations mises à jour sur les vaccins contre le virus du papillome humain (VPH) : vaccin nonavalent contre le VPH et précisions sur les intervalles minimums entre les doses dans le calendrier d'immunisation contre le VPH. http://www.healthycanadians.gc.ca/publications/healthyliving-vie-saine/human-papillomavirus-9-valent-vaccineupdate-recommendation-mises-a-jour-recommandationspapillome-humain-vaccin-nonavalent/index-fra.php.

2. Comité consultatif national de l'immunisation. Guide canadien d'immunisation: Partie 4: Vaccin contre le virus du papillome humain. Ottawa (ON): Agence de la santé publique du Canada [date de modification : 2016 juil. 07]. http://www.phac-aspc.gc.ca/publicat/cig-gci/p04-hpv-vphfra.php. 